\title{
La paradoja del poder: ¿elitización o empoderamiento colectivo?*
}

\author{
Francesco Emanuel Penaglia Vasquez \\ Centro Internacional de Economía Social y Cooperativa (CIESCOOP), Universidad de \\ Santiago de Chile, Santiago, Chile. Email: Francesco.penaglia@usach.cl
}

Resumen: La participación - entendida como la influencia e intervención de la ciudadanía en asuntos públicos y en el sistema político- requiere de mayores capacidades de acción y posicionamiento. Por lo tanto, el estudio del poder al interior de la sociedad civil es un tema central, más aun entendiendo el contexto actual de movimientos sociales y fenómenos que establecen una nueva relación entre sociedad civil y Estado. De esta forma, el siguiente artículo indaga en distintas perspectivas teóricas estableciendo la "paradoja del poder", a través de la cual la organización y coordinación de la comunidad en asociaciones, provee mayores capacidades de acción y empoderamiento de la colectividad (mayor participación); a la vez que puede generar la elitización y concentración del poder en un grupo dirigencial que no represente la totalidad de los intereses de la comunidad (menor participación). político

Palabras clave: poder, participación, elitización, sociedad civil, sistema

\section{The paradox of power: elitization or collective empowerment?}

Abstract: Participation -understood as the influence and intervention of citizenship in public issues and in the political system- requires greater action and empowerment capacity. Therefore, the study of power inside the civil society is a central topic; further, understanding the current context of social movements and phenomena that establish a new relationship between civil society and the State. Thus, the following article investigates different theoretical perspectives establishing the "paradox of power" through which the organization and coordination of the community in associations, provides more action and empowerment capacities of the collectivity (more participation); as well as it can generate the elitization and the concentration of power in a directing group who does not represent all the interests of the community (less participation).

Key words: power, participation, elitization, civil society, political system.

\section{O paradoxo do poder: elitização ou empoderamento coletivo?}

Resumo: Participação-Entende-se como a influência e intervenção da cidadania nos assuntos públicos e no sistema político. A participação requer mais ação e capacidade de empowerment. Portanto, o estudo do poder dentro da sociedade civil é um tema central; ainda mais, a compreensão do contexto atual dos movimentos sociais e fenômenos que estabelecem uma nova relação entre sociedade 
civil e do Estado. Assim, o seguinte artigo investiga diferentes perspectivas teóricas que estabelecem o “paradoxo do poder” através do qual a organização e coordenação da comunidade em associações fornece mais capacidades de ação e empoderamento das coletividade (maior participação); ao mesmo tempo, ele pode gerar a elitização ea concentração do poder em um grupo líder que não representa todos os interesses da comunidade (menor participação). político

Palavras-chave: poder, participação, elitização, sociedade civil, sistema

\section{Introducción}

Las relaciones que construye el ser humano en su condición de ser social pueden ser vistas, analizadas y estudiadas en su totalidad, como relaciones de poder. De esta forma, las relaciones de pareja, familia o amistad, laborales, organizacionales y vínculos interhumanos, se encuentran condicionados por una fuerte dinámica de poder que determina factores como lazo, liderazgo, relación y dominación, entre otros.

Evidentemente las dinámicas políticas -desde las relaciones sociales hasta la construcción del Estado- no se encuentran ajenas al análisis de poder. En esta línea, para el marxismo clásico, y con mayor énfasis en el "neo marxismo" de Antonio Gramsci, se establece que el Estado nace como un mecanismo coercitivo, que posee el monopolio del uso legítimo de la fuerza (Weber), "con aparatos necesarios (leyes, burocracia, fuerzas armadas y policía) que hacen posible el sometimiento de la población” (Garcés 2002: 2).

Gramsci sostiene que el Estado somete no sólo por la fuerza, sino también a través del establecimiento del consenso, utilizado como una de sus más importantes herramientas de dominación. De esta manera, para éste autor, el grupo hegemónico o dominante de la sociedad construye consensos socialmente aceptados o realidades "naturales” que facilitan su control. Este análisis es profundizado posteriormente por Michel Foucault, quien sostiene que el ejercicio del poder tiene una parte fundamental que es la construcción de verdades a través de discursos, señalando que "estamos sometidos a la producción de la verdad desde el poder y no podemos ejercitar el poder más que a través de la producción de la verdad” (Foucault 1980: 140). A su vez, agrega como un tercer factor (sumado al ejercicio del poder y a la construcción de verdades) una perspectiva relacionada con el derecho.

De esta forma Foucault establece una relación directa entre poder hegemónico, verdad y derecho, señalando que todo grupo dominante debe realizar producciones ideológicas como la educación, un ideal democrático y ciertas verdades (o consensos para Gramsci) que sean aceptados por toda la sociedad, creando "instrumentos efectivos de formación y de acumulación del saber, métodos de observación, técnicas de registro, procedimientos de indagación y de pesquisa, aparatos de verificación.” (Foucault 1980: 147) 


\section{Política, poder y participación}

En una perspectiva amplia, la política está relacionada con los procesos de toma de decisión y dirección de asuntos públicos, tanto en el escenario de las antiguas polis griegas como en las actuales y complejas sociedades modernas. Como ya hemos señalado, el poder es un elemento central en el análisis político, ya sea desde la teoría de las élites (Pareto, Mosca y Michels), en una concepción sociológica (Weberiana o Marxista) o desde la teoría pluralista (Dahl). Por lo que, el único modo que poseen los diversos actores de la sociedad ${ }^{1}$ para conseguir una decisión pública es a través del poder influyendo en el sistema político. ${ }^{2}$

En la búsqueda por influir en la toma de decisiones, los diversos actores llevan a cabo múltiples "luchas” o “disputas”, las que generalmente se efectúan en un marco "pacífico" llamado institucionalidad, en el que se desarrolla una competencia regulada enmarcada en un orden legitimante. Con ello el sistema político norma la discusión, estableciendo además el campo de las "ideas válidas"33 con lo que facilita la resolución de conflictos al interior del sistema, reduciendo las posibilidades de crisis estructurales (revoluciones).

Es así como las acciones políticas pueden adoptar dos mecanismos de intervención: una vía sistémica, caracterizada por el desarrollo de acciones consensuadas o luchas reguladas de actores legítimos que realizan una competencia política; o una vía extra sistémica, en la que no es posible solucionar, absorber o regular los conflictos, radicalizándose la lucha política hacia un campo de violencia expresada a través de acciones y mecanismos de presión (manifestaciones, paros, tomas y huelgas) o con crisis estructurales del sistema político (golpes militares y guerras civiles).

De esta forma, tal como señala la tabla $\mathrm{N}^{\circ} 1$, existen cuatro mecanismos de acción enmarcadas en acciones sistémicas o extra sistémicas.

\section{Tabla Nº1: Vías de acción política}

\begin{tabular}{|c|c|c|c|}
\hline \multicolumn{2}{|c|}{ Acciones sistémicas } & \multicolumn{2}{|c|}{ Acciones extra sistémicas } \\
\hline $\begin{array}{l}\text { Acciones } \\
\text { consensuadas }\end{array}$ & Lucha regulada & Violencia controlada & Crisis del sistema \\
\hline $\begin{array}{l}\text { No existen } \\
\text { posiciones } \\
\text { antagónicas por } \\
\text { tanto las acciones } \\
\text { se realizan a través } \\
\text { del acuerdo de los } \\
\text { diversos actores. }\end{array}$ & $\begin{array}{l}\text { Existen posiciones } \\
\text { divergentes y/o } \\
\text { antagónicas, pero el } \\
\text { conflicto se resuelve al } \\
\text { interior del sistema a } \\
\text { través de la posición de } \\
\text { una mayoría (política } \\
\text { representante) que decide } \\
\text { y una minoría que acepta } \\
\text { la decisión. }\end{array}$ & $\begin{array}{l}\text { Los actores aun } \\
\text { reconocen al sistema } \\
\text { como un mecanismo } \\
\text { resolutorio de } \\
\text { conflictos, sin } \\
\text { embargo ejercen } \\
\text { acciones extra } \\
\text { sistémicas para } \\
\text { presionar. }\end{array}$ & $\begin{array}{l}\text { Algunos actores no } \\
\text { reconocen al sistema } \\
\text { como el mecanismo } \\
\text { resolutorio de } \\
\text { conflictos y quiebran } \\
\text { la institucionalidad, } \\
\text { para transformarla. }\end{array}$ \\
\hline
\end{tabular}

Fuente: Elaboración Propia 
Sin embargo, el desarrollo de una de estas vías depende de tres factores que conforman la acción política de los actores: a) el ideario de los actores, el que determinará cuan distintas y divergentes son las ideas al interior del sistema, b) la capacidad de adaptación del sistema, lo que permitirá regular y resolver conflictos de mejor o peor manera y c) el poder que posee cada actor del sistema político (distribución). Por lo tanto, tal como muestra el Esquema 1.1, la conjugación de los tres elementos antes nombrados permitirá el desarrollo de acciones sistémicas o extra sistémicas. De esta manera, la capacidad de acción se transforma en la variable dependiente del poder.

\section{Esquema 1: Condicionantes de la acción política.}

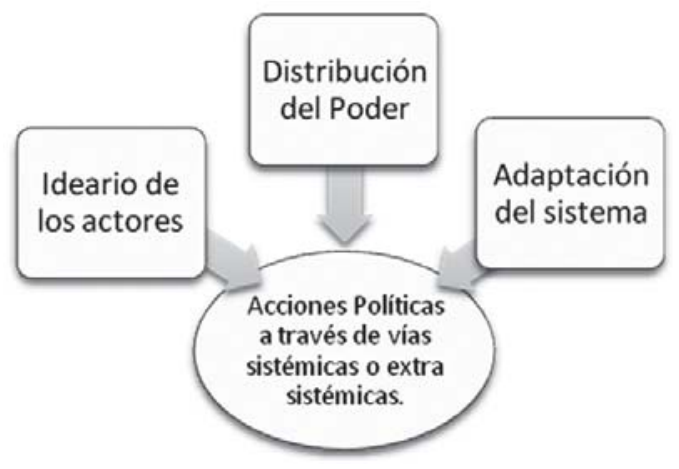

Fuente: Elaboración Propia

Como se ha señalado, el concepto de poder posee una importancia central a la hora de analizar la acción de los actores en el sistema, pero ¿qué entendemos por poder? El término asume ciertamente complejidades interpretativas; etimológicamente el concepto de poder posee en inglés y alemán un significado que en español tiene dos acepciones. Por una lado, para la Real Academia de la Lengua Española (RAE), “poder” implica la capacidad de hacer algo, pero a su vez incorpora el ejercicio de esa capacidad. Este pequeño matiz, proporciona una línea divisoria entre uso y tenencia de poder.

Por ejemplo el Estado, tiene el monopolio del uso legítimo de la fuerza (y por tanto una concentración de poder), sin embargo no siempre hace uso de sus facultades. Este matiz también se observa en el comportamiento de los individuos, en el que el poder y su uso dependen en muchas ocasiones de diversos factores asociados. De este modo, tal como señala Dahl, el poder "es una propiedad potencial, y el hecho de que sea ejercido o no depende de la presencia de determinadas circunstancias, entre ellas, por ejemplo, los recursos materiales, morales, personales y organizativos que proveen de mayor o menor capacidad de acción a los individuos” (Maíz: 6) 
Finalmente, es importante señalar que esta capacidad de poder depende tanto de factores sociales y contextuales (pertenencia a una élite, recursos, capital social); como de factores individuales, psicológicos y motivacionales. El vínculo de estos factores, condicionará tanto las capacidades del poder como el ejercicio efectivo de éste. Al respecto Dahl señala que existen un homo civicus, caracterizado por ciudadanos inactivos y conformistas y un homo politicus, conformado por ciudadanos participativos y activos. Por lo tanto, el ser humano como ser social y ser político, debe desarrollar su capacidad de poder y aplicarla haciendo uso de ésta capacidad, incrementando así sus posibilidades de intervenir e influir en los asuntos públicos.

\section{La paradoja del poder}

"El poder es una necesidad humana y social. Las personas requieren aumentar su dotación de capacidades originales para lograr sus fines y satisfacer sus deseo...El poder necesario para el Desarrollo Humano es aquel que permite aumentar la autodeterminación social del poder como un fin en sí mismo, y como un medio para el despliegue complementario de la soberanía personal real, del poder de los sistemas e instituciones, y de la definición cultural de la realidad” (PNUD 2004: 18).

Siendo o no una "necesidad humana", el poder es una elemento central en la participación y en las políticas públicas, en cuanto posibilita la negociación ${ }^{4}$ de los actores en el sistema político. Sin embargo, el poder posee dos perspectivas en algunos casos excluyentes; por un lado, el poder se construye y "potencia” mediante las relaciones sociales y la acción conjunta, por otro lado, éste se vincula al desarrollo de las capacidades individuales. De esta manera, el poder tiene un paradigma social (colectivo) y un paradigma individual, lo que se entiende como la "paradoja del poder”.

Esta paradoja está caracterizada por la necesidad de los individuos de aumentar sus capacidades para conseguir sus objetivos, lo que se consigue colectivamente; es decir, mientras más agrupadamente actúa un individuo, mayores posibilidades tiene de desarrollar sus capacidades personales y conseguir sus objetivos, lo que evidentemente supone ceder grados de autonomía y "libertad", para construir intereses conjuntos.

\section{Paradigma social del poder: El ser social}

Diversas perspectivas teóricas han desarrollado sus fundamentos, buscando demostrar la identidad social del ser humano, concepto que en la actualidad parece estar incorporado universalmente incluso por investigadores ligados a los paradigmas genéticos de comportamiento ${ }^{5}$. 
Un análisis bibliográfico de diversas corrientes de pensamiento y disciplinas de investigación demuestra que el ser humano posee un componente social que le permitió a través de su desarrollo histórico subsistir colectivamente, tanto para la caza, la procreación y el desarrollo de las necesidades básicas; como para la construcción del desarrollo cultural, valórico, religioso, político, tecnológico e histórico, entre otros. Es decir, la realidad de la raza humana es una realidad social, en la que el desarrollo y la concreción de objetivos relacionados con el poder, la política y la participación poseen necesariamente una perspectiva social (tal como constata la paradoja del poder).

El siguiente cuadro, resume algunas de las perspectivas teóricas y disciplinarias que explican la concepción del hombre como un ser social.

\section{Tabla $N^{\circ}$ 2: El ser social}

\begin{tabular}{|c|c|}
\hline Disciplina & Argumento \\
\hline $\begin{array}{l}\text { Biológica- } \\
\text { genética } \\
\text { (Darwiniana) }\end{array}$ & $\begin{array}{l}\text { El ser humano es social por factores de adaptación, predominancia y } \\
\text { sobrevivencia de la especie. El ser humano apostó a un desarrollo social que le } \\
\text { ha traído éxito hasta el momento. }\end{array}$ \\
\hline $\begin{array}{l}\text { Filosófica } \\
\text { dialéctica }\end{array}$ & $\begin{array}{l}\text { El ser humano necesita un "no ser" y objetos fuera de sí para comprender la } \\
\text { realidad, la existencia sólo es posible en relación a sujetos y objetos externos. }\end{array}$ \\
\hline $\begin{array}{l}\text { Filosófica } \\
\text { Aristotélica }\end{array}$ & $\begin{array}{l}\text { Desde una mirada aristotélica los hombres han sido engendrados por otros } \\
\text { hombres a quienes deben su existencia. Desde el mismo apareamiento hasta el } \\
\text { aprendizaje }\end{array}$ \\
\hline $\begin{array}{l}\text { Sociológica: } \\
\text { Durkheim }\end{array}$ & $\begin{array}{l}\text { Todo existe socialmente, nosotros descubrimos mediante el aprendizaje "desde } \\
\text { el sistema de signos que utilizo para expresar mis pensamientos" hasta las } \\
\text { motivaciones y acciones son parte de un contexto social. }\end{array}$ \\
\hline $\begin{array}{l}\text { Económico } \\
\text { Smith y Marx }\end{array}$ & $\begin{array}{l}\text { Sin intercambio entre individuos, cada uno debiese satisfacer sus propias } \\
\text { necesidades. }\end{array}$ \\
\hline $\begin{array}{l}\text { Visión política } \\
\text { de la sociedad: } \\
\text { Hannah Arendt. }\end{array}$ & $\begin{array}{l}\text { E1 ser humano forma lazos de unión, "estar juntos" en, comunidades y } \\
\text { construcciones políticas como proyección de una vida familiar-comunitaria }\end{array}$ \\
\hline
\end{tabular}

Fuente: Elaboración Propia

De esta manera, ya sea por razones económicas, sociales, antropológicas o biológicas, el ser humano actúa colectivamente para satisfacer su amplio espectro de necesidades. A raíz de ello, y retomando nuestra perspectiva de poder, los individuos poseen mayores capacidades, coordinación, influencia y evidentemente poder, actuando organizadamente. Esto permite estudiar a las organizaciones de la sociedad civil, como fuentes de poder, las que relacionan a individuos bajo un mismo objetivo, potenciando sus capacidades de éxito (en comparación a su acción individualizada). 


\section{El Paradigma individual del Poder}

"Desgraciadamente, el mismo estado social que tan necesarias hace las asociaciones en los pueblos democráticos, las hace más difíciles que en ningún otro...Cuando varios miembros de una aristocracia desean asociarse, lo consiguen fácilmente. Comoquiera que cada uno de ellos aporta una gran fuerza a la sociedad, el numero puede ser muy pequeño y cuando los socios son pocos les resulte fácil conocerse, comprenderse y establecer reglas fijas para su acción. No se encuentra la misma facilidad en las naciones democráticas, donde siempre es preciso que los asociados sean numerosos para que la asociación posea cierta potencia” (Tocqueville 1840: 97).

Una segunda perspectiva es la desarrollada por la escuela de "las élites" (Pareto, Mosca y Michels), la que sostiene que la "tendencia a la oligarquización” es una dinámica natural de las organizaciones humanas. $\mathrm{Al}$ respecto, Robert Michels señala que las organizaciones y agrupaciones se disgregan entre una minoría conformada por líderes o directivos y una mayoría de dirigidos compuesta por la base organizacional. Por otro lado, esta perspectiva teórica constata que mientras más grande y extensa es la organización (en cuanto a objetivos, acciones y envergadura), más poder se concentra en los líderes, por lo tanto existe una directa proporción entre el tamaño organizacional y la concentración del poder.

La relación entre estos factores se explica en cuanto "toda organización implica especializacion y responsabilidad de los lideres, los cuales se hacen expertos en conducir a las masas. Asi, la democracia termina convirtiéndose en una forma de gobierno de los mejores: en una aristocracia” (Bolivar: 398). Con ello el poder no recaería en el colectivo, puesto que la tendencia natural de la organización sería la creación de jerarquías o liderazgos que no representan al conjunto de los asociados a la organizacion, sino a sus particulares intereses.

Este argumento extrapolado a un analisis macro, concluye que la historia social está marcada por la lucha entre élites, las que en muchos casos toman el poder manipulando al pueblo. Es así, como la capacidad "pacifica” o "institucional” del sistema, busca generar estabilidad a través de la renovacion paulatina de las élites, cooptando los movimientos populares e incorporando a su lideres a la politica "oficial". De no ocurrir ello, el sistema colapsaría generándose revoluciones (como ha ocurrido a lo largo de la historia), las que a juicio de estos autores no serian otra cosa más que el cambio de élites ${ }^{6}$. De esta manera, tal como señala Pareto "la lucha y la circulacion de las élites es la esencia de la historia; por lo tanto, los levantamientos populares no siempre tienen conseciencias positivas para el pueblo, sirven sólo para facilitar la caida de la vieja élite y el surgimiento de la nueva" (Bolivar: 390)

Con ello estos teóricos suponen que las organizaciones de la sociedad civil, no son otra cosa que soportes de caudillos o liderazgos, que tienen obje- 
tivos particulares, por lo tanto detrás de la asociatividad y la organización del individuo se esconde siempre la dominacion y el sometimiento. Esto entrega una perspectiva contraria a quienes relacionan el poder con la capacidad colectiva, asociativa y organizativa de la sociedad, que supone que los individuos al vincularse con otros incrementan su poder y sus capacidades de desarrollo.

En esta misma perspectiva, el sociólogo peruano Martín Tanaka señala que la comunidad -en un sentido tocquevilliano- no existe, puesto que es imposible hablar de individuos con intereses colectivos armónicos. Este autor sostiene que sólo se podria hablar de "comunidades" en zonas aisladas y pequeñas, generalmente pobres, que posean poca intervencion de agentes externos, las que en ciertas ocasiones logran desarrollar acciones colectivas cohesionadas con sentidos comunitarios. Sin embargo, en las sociedades modernas y en las grandes urbes se encontrarían "subgrupos altamente diferenciados, con conflictos, relaciones de dominio y subordinación, distintos grados de poder e influencia, orientaciones sociales y políticas diversas” (Tanaka 2001: 12)

Por lo tanto, toda organización o movimiento social es sujeto de luchas internas, dinámicas de poder y dominaciones; por ende los liderazgos que emergen y controlan un movimiento (como señalan los teóricos “elitistas") no serían otra cosa que la representación de intereses particulares o de actores con más recursos (económicos, intelectuales, carismáticos, redes, etc.) y de poder.

De esta forma "se asume por lo general que estos personajes (dirigentes) representan y dirigen a la comunidad (sin embargo) la comunidad como tal no existe, de allí que estos dirigentes difícilmente puedan hablar en nombre de la comunidad. Más adecuado sería pensar que en realidad son dirigentes y representantes de un sector de una comunidad que tiene por definición múltiples maneras de expresarse”. (Tanaka 2001: 14)

Para Tanaka existe un problema frecuente y recurrente en la forma de observar y constituir las organizaciones comunitarias, sociales y políticas, replicando un modelo piramidal (ejemplificado en el siguiente esque$\mathrm{ma}$ ), el que supone una comunidad con intereses comunes en la base, con unidad de acción y expresión la que es captada por dirigentes y líderes que canalizan, representan y dan forma a las necesidades de la comunidad.

\section{Esquema $N^{\circ} 2$ : Modelo Piramidal}

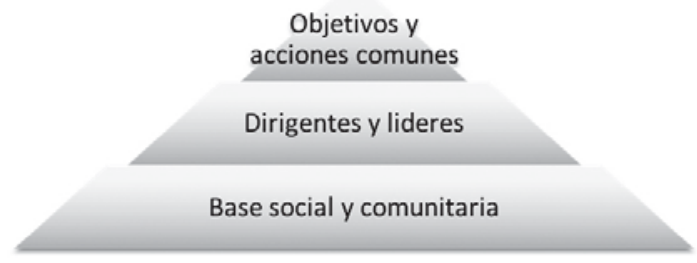

Fuente: elaboración propia 
De esta manera, tanto la perspectiva de las élites como la concepción de Tanaka, suponen que las organizaciones, ya sea por las dinámicas dirigentes-dirigidos, por las pugnas internas de poder e influencia o por los sesgos que supone la representatividad y el modelo piramidal, poseen dinámicas de dominación interna.

Por lo tanto, contrario a la perspectiva social del poder (que enfatizaba que la creación de instancias coordinadoras de la sociedad permitía “construir puentes sociales” entre las organizaciones empoderando a la sociedad civil y a los individuos organizados), la perspectiva social de las élites señala que las creación de estas instancias sólo contribuiría a generar relaciones de dominación, hegemonía y cooptación, en las que se genera necesariamente un grupo líder que conduciría el destino de la organización. De esta forma, la unión y coordinación entre organizaciones sociales no beneficiaría a nadie más que al grupo hegemónico, socavando el desarrollo individual del poder, sometiendo a los organizados a una categoría de masa, objeto y no sujeto de los cambios sociales.

A conclusiones similares llegan algunos teóricos argentinos desde la perspectiva del capital social, cuando señala que "a medida que nos encontramos con estructuras sociales más cerradas, caracterizadas por relaciones de alto grado de interdependencia entre los individuos, se hace posible controlar los comportamientos oportunistas, disminuir el riesgo en los intercambios y, con ello, darle continuidad a las confianzas y reciprocidades" (Forni, Siles, Barreiro 2004:7). Por lo tanto, el valor organizativo estaría en la heterogeneidad, micro asociatividad y sociedades policéntricas.

\section{Reflexiones finales}

En síntesis, existen, a lo menos, dos concepciones o líneas analíticas que ponderan de manera divergente las dinámicas de poder, representadas en lo que se ha denominado "paradoja del poder", vinculadas a dos visiones opuestas sobre las asociatividades, organizaciones, redes y emprendimientos de las comunidades y la sociedad. En la siguiente tabla se resumen las ideas-fuerza presentes en las perspectivas social e individual de la paradoja del poder (Tabla $\mathrm{N}^{\circ} 3$, página siguiente). 


\section{Tabla N³: Síntesis conceptual}

\section{Perspectiva social de poder}

Perspectiva Individual de poder

Premisa 1: Las organizaciones sociales al Premisa 1: Las instancias coordinadoras de la coordinarse, asociarse y unirse, poseen mayor poder, con lo que se incrementan sus capacidades de influir en el sistema político y las políticas públicas. sociedad civil y de organizaciones comunitarias, esconden mecanismos de dominación, hegemonía y cooptación. El poder que se genera en las uniones se acumula y concentra en un solo grupo.

Premisa 2: Los individuos poseen mayores posibilidades de desarrollarse vinculándose Premisa 2: Los individuos que se empoderan son con otros, por tanto se incrementa la los que dominan y lideran la organización, por participación comunitaria canalizada a través de las organizaciones a las que se encuentran asociados. tanto el resto de los asociados son utilizados y subordinados. Los individuos no se empoderan ni se desarrollan en el colectivo, sino más bien son sometidos por un interés general que no los representa.

Por lo tanto, establecida la paradoja del poder, será el resultado de futuras investigaciones y estudios definir e identificar, cuál de estas dos expresiones teóricas explica de mejor manera los fenómenos sociales.

Esto será fundamental para comprender los procesos orgánicos que vive la sociedad civil, indagando en los factores críticos que posibiliten el real empoderamiento y democratización de la sociedad, convergiendo finalmente a una ciudadanía cada vez más autónoma, capaz y dueña de su destino. 


\section{Notas}

*El presente artículo extrae algunos elementos del marco teórico, de la tesis de titulación del autor, denominada: “¿Poder colectivo o elitización dirigencial?. El caso de las Uniones Comunales de Maipú”.

${ }^{1}$ Se entiende por actores del sistema a Ciudadanos, élites, empresas, partidos políticos, sindicatos, organizaciones comunitarias, grupos religiosos, think tank, comunidades epistémicas, grupos económicos, grupos de presión, grupos de interés y la sociedad civil en general.

${ }^{2}$ Ésta definición de poder está relacionada a otras definiciones encontradas en la literatura política, entre las que se pueden mencionar: "habilidad o la capacidad de hacer que sucedan cosas"; "generar intencionalmente efectos" (Russell 1938); "capacidad de un actor para producir resultados exitosos" (Wrong 1979: 1); "capacidad de un actor de conseguir que otro haga lo que de otro modo éste no haría” (Dahl 1957); "los medios presentes de obtener algún bien futuro aparente" (Hobbes 1979: I.10) y "la probabilidad de imponer la propia voluntad... aún contra toda resistencia” (Weber 1964: 43).

${ }^{3}$ Explicadas anteriormente en un sentido gramsciano como consenso o en la perspectiva de Foucault como verdades, las ideas validadas son el resultado de los equilibrios políticos y las correlaciones de fuerzas consideradas legítimas. Por ejemplo: "el mercado es el mejor asignador de recursos”, es un ideario que compartían los candidatos presidenciales (proceso electoral de 2010-2011) desde el "Juntos Podemos" hasta "La Alianza por Chile”, moviéndose el campo de la discusión únicamente en el grado de regulación.

${ }^{4}$ Elemento central de toda negociación es que los actores que participan en ella posean fuerzas similares. Si existen grandes desigualdades entre los actores las decisiones hegemónicas predominarán, sin que exista un costo político, social, moral o económico, dependiendo los grupos minoritarios de la voluntad de los hegemónicos para aceptar e incluir sus propuestas.

${ }^{5}$ Investigadores como los doctores Robert O. Kurzban y James H. Fowler han realizado estudios genéticos y experimentos con gemelos para demostrar que el comportamiento humano depende de factores biológicos como la serotonina; reconocen igualmente que el ser humano depende en gran medida de factores contextuales, socioculturales e históricos (Seminario: "Legado intelectual de Darwin en el siglo XXI”. Santiago, Chile, 27 de julio del 2009, Casa Piedra).

${ }^{6}$ Tal sería el caso de la Revolución Francesa, que permitió el ascenso al poder de la emergente burguesía, el caso de las guerras de independencia latinoamericanas o el caso de algunas revoluciones socialistas que generaron clases dirigenciales (que independiente de factores como el bloqueo, la guerra fría, las guerras mundiales, invasiones, intervenciones, etc.) se perpetuaron en el poder y en múltiples ocasiones no representaron los intereses de la clase proletaria. 


\section{Bibliografía}

Acuña, Eduardo; Nuñez, Alejandra; y Radrigán, Mario, (2003), Un marco conceptual para el estudio de la participación, en UNIRCOOP Reflexiones sobre el cooperativismo y el asociativismo, Vol. 1, Editado por: IRECUS, Facultad de Administración, Universidad de Sherbrooke, Quebec.

Arendt, Hannah (1997), ¿Qué es la política?, Ediciones Paidós I.C.E de la universidad autónoma, Barcelona.

Bengoa, José (2006), La comunidad reclamada, Editorial Catalonia LTDA, Santiago.

Bolívar, Rosendo (2002), “La teoría de las elites en Pareto, Mosca y Michels”. Iztapalapa.

Bourdieu, Pierre (2008), Capital cultural, escuela y espacio social. Siglo XXI ediciones. Argentina

Delamaza, Gonzalo (2010), Construcción democrática, participación ciudadana y políticas públicas en Chile, s.d

Ídem (2005), Tan lejos tan cerca. LOM ediciones. Santiago

Delamaza, Gonzalo; Flores, Daniel, ed, (2009), Gestión Municipal Participativa. Construyendo democracia cotidiana, Editorial Carla Palma, Santiago.

Durkheim, Emile (1997), “Las reglas del método sociológico”, Fondo de Cultura Económica, Mexico D. F.

Engels, Federico (2006), El Origen de la familia, la propiedad privada y el Estado, Ediciones de la revolución ecuatoriana.

Foucault, Michel (s.f), “Microfisica del poder”. Sd.

Ídem (2002), Vigilar y Castigar, Ediciones siglo 21. Argentina.

Forni, Pablo; Siles, Marcelo; Barreiro, Lucrecia (2004), “¿Qué es el Capital Social y cómo Analizarlo en contextos de Exclusión Social y Pobreza?”. Research Report

GarcésS, Mario (2001), Democracia y poder local, Eco educación y comunicaciones, Santiago

Garretón, Manuel Antonio (s.f), Cambios sociales, actores y acción colectiva en America Latina, (s.d)

Gramsci, Antonio (1988). “Antología, selección de traducciones y notas 
de Manuel Sacristán”, Siglo xxi editores. España 1988

Harnecker, Marta (2007), Democracia y Socialismo, Escuela Sociológica Universidad Central de Venezuela.

Jung, C. G. (1970), Arquetipos e inconsciente colectivo, ediciones Paidós Ibérica. Barcelona.

Lagroye, Jaques (1994), Sociología Política, Editorial Fondo de Cultura Económica.

Lechner, Norbert (s.f), La democratización en el contexto de una cultura postmoderna, S.d.

Lucas, Antonio; García, Ángela, ed (2001), Formación para la Participación Ciudadana, Grupo Editorial Lumen. Buenos Aires.

Maiz, Ramón (s.f), “Poder, Legitimidad y Dominación”. S.d.

Marx, Karl (2006), Manuscritos Económicos y Filosóficos de 1844, Editorial Colihue Clásica, Buenos Aires.

Ídem (s.f), Prologo a la contribución a la critica económica, s.d.

Mujica, Dolores (2008), Los Cordones Industriales. Clase Contra Clase, s.d

Pereda, Carlos; Prada, Miguel; Actis, Walter (2003), Iinvestigación Acción Participativa: Propuesta para un ejercicio activo de la ciudadanía. Colectivo IOÉ, Madrid.

Pinto, Julio (2007), Introducción a la Ciencia Política, Universidad de Buenos Aires, Buenos Aires.

PNUD (2000). Informe de Desarrollo Humano Chile 2000: Más Sociedad para Gobernar el Futuro. Programa de Naciones Unidas para el Desarrollo, Santiago de Chile.

Ídem, Informe de Desarrollo Humano en Chile (2004), El Poder: ¿para qué y para quién?, Programa de Naciones Unidas para el Desarrollo, Santiago de Chile.

PNUD- FOCUS (2006), Estudio Comparativo del Sector sin Fines de Lucro, John Hopkins Univeristy, Programa de Naciones Unidas para el Desarrollo, Santiago de Chile.

Rodríguez, M. Angélica, Bello, Daniela (2002), participación, ciudadanía y democracia local, Eco educación y comunicaciones, Santiago. 
Sabatini, Francisco (1998), Participación y localidad: problemas, conflictos y negociación. FLACSO, Chile.

Salazar, Gabriel (1998), Capital social constante y capital social variable, Archivo Chile, s.d.

Ídem (1999), Ciudadanía e historia oral, Archivo Chile, S.d.

Ídem (s.f), Descentralización y sinergia histórica local: Fracasos y desafíos s.d.

Salazar, Gabriel; Pinto, Julio (1999), Historia Contemporánea de Chile Vol. I, Lom ediciones, Santiago.

Ídem (1999), Historia Contemporánea de Chile Vol. II, Lom ediciones, Santiago.

Serrano, Claudia; Vicherat, Daniela (2000), ¿Qué motiva a la gente a actuar en común? Estudio sobre participación social en la población Malquías Concha comuna de La Granja , CIEPLAN, Santiago.

Tanaka, Martín (2001), “Participación popular en las políticas sociales” Instituto de estudios peruano, S.d

Tocqueville, Alexis de (1985), “La democracia en América” Vol. I y II, Alianza Edit. Barcelona.

Valle, Josep M. (s.f), ciencia política. Parte III. La política en el Estado: elementos constitutivos, instituciones, formas de gobierno. S.d

Varela, Sergi (1997), Estudio de la relación entre el espacio simbolico urbano y los procesos de identidad social, Revista de Psicología Social, 12, 17-30, Barcelona.

Villasante, Tomas (2002), Sujetos en Movimiento. Redes y procesos creativos en la complejidad social, Editorial Nordan-Comunidad, Montevideo.

Weber, Max (s.f), “El Político y el científico”, s.d.

Recibido: 15.09.2011

Aceptado: 25.07.2012 